\title{
COLD, WARM, AND COMPOSITE (COOL) COSMIC STRING MODELS
}

\author{
Brandon Carter \\ Département d'Astrophysique Relativiste et de Cosmologie, C.N.R.S., \\ Observatoire de Paris, 92195 Meudon, France. \\ (1993 preprint, submitted to Nucl. Phys. B.)
}

\begin{abstract}
The dynamical behaviour of a cosmic string is strongly affected by any reduction of the effective string tension $T$ below the constant value $T=m^{2}$ say that characterizes the simple, longitudinally Lorentz invariant, Goto Nambu string model in terms of a fixed mass scale $m$ whose magnitude depends on that of the Higgs field responsible for the existence of the string. Such a reduction occurs in the standard "hot" cosmic string model in which the effect of thermal perturbations of a simple Goto Nambu model is expressed by the formula $T^{2}=m^{2}\left(m^{2}-2 \pi \Theta^{2} / 3\right)$, where $\Theta$ is the string temperature. A qualitatively similar though analytically more complicated tension reduction phenomenon occurs in "cold" conducting cosmic string models where the role of the temperature is played by an effective chemical potential $\mu$ that is constructed as the magnitude of the phase $\varphi$ of a bosonic condensate of the kind whose existence was first proposed by Witten. The present article describes the construction and essential mechanical properties of a category of "warm" cosmic string models that are intermediate between these "hot" and "cold" extremes. These "warm" models are the string analogues of the standard Landau model for a 2-constituent finite temperature superfluid, and as such involve two independent currents interpretable as that of the entropy on one hand and that of the bosonic condensate on the other. It is surmised that the stationary (in particular ring) equilibrium states of such "warm" cosmic strings may be of cosmological significance.
\end{abstract}




\section{Introduction: the importance of longitudinal Lorentz symmetry break- ing.}

As a model for the representation of cosmic string behaviour, i.e. for the description at a macroscopic level of the phenomen whose underlying mechanism is that of a vortex defect of the vacuum (in a field theory with spontaneous symmetry breaking), the simplest possibility is specifiable by an variation principle of the well known GotoNambu type, which means that the internal state of its 2-dimensional world sheet is locally Lorentz invariant: with respect to an internal rest frame whose choice is (as an expression of the invariance property) entirely arbitrary the mass-energy density per unit length $U$ and the associated string tension $T$ will actually be equal, being given in units with $\hbar=c=1$, simply by

$$
U=T=m^{2}
$$

while the dynamical motion of such a string will be governed by an action given as the integral over the string world sheet of a scalar Lagrangian function $L$ of the trivially simple form

$$
L=-m^{2},
$$

where $m$ is a fixed mass scale which (as was pointed out by Kibble in the earliest discussion of the potential cosmological importance of the phenomenon ${ }^{[1]}$ ) may be expected to be of the same order of magnitude as the mass scale characterising the Higg's boson responsible for the spontaneous symmetry breaking, the precise value (as derived by working out the corresponding Nielsen Olesen ${ }^{[2]}$ type equilibrium state) being dependent on the details of the particular underlying field theory that is supposed to be relevant. In most of the early discussions of the subject it was postulated that the strings under consideration were of the "heavyweight" variety for which the relevant symmetry breaking was that of grand unification, as characterised by $\mathrm{Gm}^{2} \approx 10^{-6}$, but (as has recently been shown explicitly by Peter ${ }^{[3]}$ ) it is also possible to envisage "lightweight" cosmic string formation for which the relevant symmetry breaking is that of electroweak unification, as characterised by $G m^{2} \approx 10^{-32}$.

A very important consequence of the longitudinal Lorentz symmetry condition expressed by (1.1) is that an isolated string loop of such a Goto-Nambu type has no stationary equilibrium state and so must oscillate until all its energy has been lost by gravitational radiation. However, for a generic string model, in which this special local symmetry will be broken, such an energy loss process can not go through

to completion due, as was first clearly pointed out by by Davis and Shellard ${ }^{[4][5][6]}$, to 
the consequent existence of stationary equilibrium states. The recognition of this effect immediately leads to the question - which does not arise in the pure Goto-Nambu case - of whether a cosmological mass density excess $\Omega \gg 1$ would not have been produced by the formation of a distribution of stationary loop relics, at least ${ }^{[4][5][6]}$ for strings of the "heavyweight" variety characterised by $G m^{2} \approx 10^{-6}$, which makes it possible to $\operatorname{argue}^{[7][8]}$ that strings of the "lightweight" variety characterised by $G m^{2} \approx 10^{-32}$ are more likely (or less unlikely) than the "heavyweight" variety to have played a real role in the evolution of our universe.

It is to be remarked that even before the key point was made by Davis and Shellard ${ }^{[4][5][6]}$ it had already been pointed out that string loops might be maintained in equilibrium by a global as opposed to local mechanism, namely that of magnetic support ${ }^{[9]}$, in which the tendency to contraction due to the local string tension $T$ is balanced by the the effect of a globally extended dipolar magnetic field due to an electromagnetically coupled current in the loop. It was however recognised at the outset ${ }^{[9]}$ and agreed in subsequent discussions ${ }^{[10][11][12]}$ that (taking account of the smallness of the electromagnetic coupling constant) such an electromagnetic support mechanism was unlikely to be sufficiently strong to be of practical importance. The subsequent discussions gave rise nevertheless to a certain amount of confusion due to their failure to clearly distinguish this essentially global magnetic support mechanism (due to the long range effect of the external electromagnetic field arising from the charge coupled current) from the qualitatively different mechanism (which can also be considered to account for the phenomenon of current saturation, and which operates even in the absence of any electromagnetic coupling) whereby the local string tension is reduced by the mechanical effect of the current in the immediate neighbourhood of the vortex core. This confusion was embodied in the use of the potentially misleading term "spring" to describe states in which the effective tension was reduced to zero so as to allow static equilibrium without an external supporting force. More detailed examination ${ }^{[13][14]}$ of the local effect of the current appeared to confirm that in certain models the string tension $T$ actually could in principle reach zero and even negative values, but it was pointed out that in a local (as opposed to global) string state negative tension automatically implies instability ${ }^{[15]}$ and it has been shown more recently using improved numerical methods ${ }^{[16][17]}$ that although it can be considerably reduced, the local string tension $T$ will in fact remain strictly positive in the all the kinds of model that have been examined so far.

However that may be, the question of whether purely magnetically sup- 
ported equilibrium states can be of of any practical significance (and of whether local "spring" states can exist at all) was effectively relegated to obsolescence as far as cosmological implications are concerned by the observation of Davis and Shellard ${ }^{[4][5][6]}$ that there is a fundamentally different, essentially local rather than global support mechanism that is is quite independent of electromagnetic coupling and that will in any case be much more effective, namely the centrifugal effect that can operate whenever the local longitudinal Lorentz symmetry (1.1) is violated so as give a strict inequality,

$$
T<U
$$

since this is all that is required to allow existence of centrifugally supported equilibrium states (of which the simplest ${ }^{[18][19]}$, though not the only type ${ }^{[20][21]}$, are circular ring configurations) which are characterised by a subluminal longitudinal running speed vi1 that is given quite generally by

$$
v^{2}=\frac{T}{U}
$$

\section{Lorentz symmetry breaking by Witten's "cold" current mechanism.}

The development that originally raised the question of the cosmological implications of longitudinal Lorentz symmetry breaking and the consequent existence of centrifugally supported equilibrium states was the demonstration by Witten ${ }^{[22]}$ that for field theories of a kind only slightly more complicated than that on which Kibble's original analysis was based, the core of the vortex defect of the vacuum may be inhabited by a boson condensate of the type familiar in ordinary superfluid theory with the implication that it may support a corresponding dissipationless current. At a macroscopic level the resulting "superconducting" (or to be technically more precise "charged superfluid") string behaviour will be describable by a type of model that is specifiable ${ }^{[18][23]}$ by replacing the degenerate (constant) Lagrangian (1.2) by a function of a variable mass, $\mu \approx m$, obtained as the magnitude of an effective energy-momentum covector $\mu_{\rho}$ that is itself constructed from the gradient of a scalar phase field $\varphi$.

In cases for which no coupling to any external field need be taken into account, the energy-momentum covevctor will be given simply by

$$
\mu_{\rho}=\widetilde{\nabla}_{\rho} \varphi
$$

where the $\widetilde{\nabla}_{\mu}$ denotes the worldsheet projected gradient operator ${ }^{[18][23]}$ as given by

$$
\widetilde{\nabla}_{\rho}=\eta_{\rho}{ }^{\sigma} \nabla_{\sigma}
$$


in terms of the fundamental ${ }^{[18][23]}$ tensor $\eta_{\rho}{ }^{\sigma}$ (whose definition is given later on in section $5)$.

The form of Lagrangian function

$$
L=L(\mu), \quad \mu^{2}=-\mu_{\rho} \mu^{\rho}
$$

that is to be used in place of (1.2) will remain unaffected by allowance for charge coupling to an external electromagnetic field, such as was postulated in Witten's original discussion $^{[22]}$, the only effect of such a coupling, when relevant, being to modify the defining formula for the energy momentum covector $\mu_{\rho}$ by the requirement that the appropriate gauge covariant differentiation operator $D_{\rho}$ should be used instead of $\nabla_{\rho}$. Such secondary external coupling effects will not however be considered in the present discussion, partly because they would divert attention from the new concepts that will be presented, and partly because they no longer seem quite to be of quite such dominant importance as was once supposed.

Actually, although great importance was attributed to charge coupling effects in the earliest discussions of the Witten mechanism ${ }^{[9]}$ it has become apparent since the work of Davis and Shellard ${ }^{[4]}$, and particularly since the recent work of Peter ${ }^{[17]}$ that they are relatively less important than the purely mechanical effects to which our attention will be restricted in the present work: the smallness of the electromagnetic "fine structure" coupling constant $e^{2}=1 / 137$ justifies its neglect, at least as a lowest order approximation, in a wide range of circumstances, which in the context of conducting strings has the significant technical advantage of ensuring that there is no need to worry about the subtle infrared divergence problems (violation of the locality postulate on which a string description in the strict sense is based) that would otherwise arise. Subject to this condition, i.e. assuming either the actual absence or the effective negligibility of any electromagnetic coupling, Peter has carried out accurate numerical calculations (superseding earlier more approximate investigations ${ }^{[14]}$ ) of the equation of state specifying the non-linear Lagrangian to be used in (2.3) on the basis of a simplified toy bosonic field theory ${ }^{[16]}$ (with two complex scalar fields but just one gauge boson) whose complexity is midway between the one originally used by Kibble ${ }^{[1]}$ for the non conducting case (with just one complex scalar field and one vector gauge boson) and the one originally proposed by Witten ${ }^{[22]}$ (which had not only two complex scalar fields but also two independent vector gauge bosons).

Although Witten originally raised the question of conductivity in cosmic strings on the basis of a model that was too highly simplified to be realistic, a large 
number of other conceivable (fermionic as well as bosonic) "superconduction" mechanisms have since been suggested by many authors in less artificially idealised models (of which one of the most realistic is perhaps the kind recently discussed by Peter ${ }^{[3]}$ within the framework of the modified electroweak unification theory that has been proposed by Fayet ${ }^{[24]}$.) The existence of such a rich range of possibilities suggests that far from being exceptional, "superconductivity" of one kind or another is likely to be a generic feature of cosmic strings if they exist at all, or to put it another way, the postulate of its total absence would seem to be a severely restrictive condition to impose on any would-be realistic cosmic string forming field theory.

Unless (conceivably as an application of a sufficiently strong version of the anthropic principle ${ }^{[25]}$ ) a specially contrived non superconducting string forming theory actually does apply, the expected currents will determine corresponding conserved quantities ${ }^{[18][19][23]}$ on any closed string loops that may be formed, which - provided the relevant length scales are large enough for quantum tunnelling to be negligible so that a purely classical description remains valid - will exclude the possibility of their complete destruction by radiative energy loss so that the stationary relic formation process first envisaged by Davis and Shellard ${ }^{[4]}$ will inevitably occur. As was remarked in the preceeding section, for "heavyweight" (G.U.T. mass scale) strings as characterised by $G m^{2} \approx 10^{-6}$, simple dimensional considerations ${ }^{[4][5][6][7][8]}$ lead to the prediction of such an extremely large cosmological mass density excess $\Omega \gg 1$ that even if the efficiency of the relic loop formation were very low it would still be hard to obtain a realistic scenario with $\Omega \approx 1$ unless the classical description is invalidated at some stage. As a way of saving the scenario of galaxy formation by heavyweight cosmic string formation, Davis and Shellard suggested ${ }^{[4][5]}$ that it might after all be possible to get rid of nearly all the unwanted relic loops by quantum tunnelling to non-conducting Kibble type states. However although such tunnelling processes will no doubt have some effect, it is evident that the corresponding decay timescales will be very sensitively dependent on the length scales and wavelengths that are involved, and hence indirectly on the values of the classically conserved numbers by which the loops are characterised. The random processes by which the loops are formed will presumably give rise to widely scattered values for the corresponding conserved numbers, so that, although many or even most may fall in a range for which the corresponding tunnelling timescales are cosmologically short, the existence of a systematic mechanism to prevent even a small fraction from being stable over cosmologically long timescales is not at all obvious. The implication is that the viability of the heavyweight string scenario is much more seriously threatenned by 
the loop preservation mechanism of Davis and Shellard than these authors themselves originally realised.

On the other hand, although some rather miraculous (e.g. anthopic) deus ex machina process would seem to be needed to restore the credibility of string formation scenarios with $G m^{2} \approx 10^{-6}$, it is nevertheless to be emphasized that there is no such difficulty for lightweight string scenarios as characterised by the electroweak value $G m^{2} \approx 10^{-32}$ which appears on the basis of plausible assumptions ${ }^{[7][8]}$ to be just high enough to allow the ensuing distribution of relic loops to provide a cosmologically significant mass distribution, $\Omega \approx 1$, in the form of what would presumably be perceived as a cold dark matter contribution. 


\section{The standard "hot" string model.}

The main purpose of the present work is to draw attention to, and provide a mathematical framework for analysing, another effect of a different nature to those discussed in the previous sections, which also effectively contributes to longitudinal Lorentz symmetry breaking and therefore enhances the efficiency of the loop preservation phenomenon whose potential cosmological importance has been underlined above. The effect in question is that of stochastic, and more particularly thermal excitation not just of internal excitation modes within the string, whose energy thresholds may conceivably be too high for them to be important, but more particularly of the extrinsic perturbation modes whose potential relevance can not be doubted, since their existence is already implicit in any string description at all, including the ultra simple GotoNambu model characterised by (1.2) as well as the less degenerate models characterised by $(2.3)$.

Even in the case of a model of the simple non-superconducting type originally proposed by Kibble ${ }^{[1]}$, a more accurate treatment would require allowance for the excitation of shortwavelength modes which would cause deviations from the simplified description based just on the constant Goto Nambu action (1.2). If the spectrum is thermal and if the only modes that are excited are extrinsic transverse displacements of the string worldsheet itself then a simple heuristic argument lead me to the prediction $^{[26]}$ that the motion will still be describable by a variation principle, but one in which the constant (1.2) is replaced by an effective Lagrangian that is a function of the relevant surface density current of entropy, $s^{\rho}$ say, whose existence evidently breaks the longitudinal Lorentz symmetry by specifying a corresponding preferred thermal rest frame. When expressed in energy units (such that Boltzmann's constant is set to unity, i.e. $\mathrm{k}=1$ ) the explicit analytic form of the predicted Lagrangian is given by the simple expression

$$
L=-m^{2}\left\{1+\frac{3 s^{2}}{2 \pi m^{2}}\right\}^{1 / 2}, \quad s^{2}=-s^{\rho} s_{\rho}
$$

where the scalar $s$ is interpretable as the surface density of entropy in the thermal rest frame. In the application of the variation principle the surface current vector $s^{\rho}$ is to be varied not quite freely but, subject (as discussed in detail in the Section 5) to a constraint ensuring that its surface divergence, $\widetilde{\nabla}_{\rho} s^{\rho}$ vanishes.

The resulting mechanical behaviour in this standard "hot" cosmic string model is describable in terms of a thermal rest frame surface energy $U$ and a corre- 
sponding string tension $T$ that are given by

$$
U=\frac{m^{4}}{T}=-L
$$

The qualitative conclusion that the product $U T$ remains constant is not dependent on the supposition that the excitation "noise" spectrum is strictly thermal, and has been confirmed by a more detailed and explicit calculation of the effect of small "wiggles" by Vilenkin ${ }^{[27]}$. The "constant product" equation of state (3.2) can be shown to imply direct integrability of the dynamic equations not only in a flat background ${ }^{[26]}$ but also for the case of stationary equilibrium in a Kerr black hole background ${ }^{[19]}$, the underlying reason for all these exceptionally convenient properties being that this particular kind of equation of state is uniquely characterise by equality of the extrinsic and longitudinal characteristic speeds $c_{\mathrm{E}}$ and $c_{\mathrm{L}}$ as given ${ }^{[15]}$ by

$$
c_{\mathrm{E}}^{2}=\frac{T}{U}, \quad c_{\mathrm{L}}^{2}=-\frac{d T}{d U}
$$

It is to be remarked that behaviour characterised by the same "constant product" equation of state $(3.2)$ can also be shown ${ }^{[18]}$ to arise in the physically very different and rather artificial context of Nielsen's Kaluza Klein mechanism ${ }^{[28]}$.

In the natural thermal case with which we are concerned here, the corresponding local temperature of the string, which it is convenient to denote by $\Theta$ (to avoid confusion with the tension $T$ ) will be obtainable from (3.1) by application of the standard defining relation

$$
\Theta=\frac{d U}{d s}
$$

which leads to the relation

$$
\frac{2 \pi \Theta^{2}}{3 m^{2}}=1-\frac{T^{2}}{m^{4}}
$$

It can be seen that starting from the longitudinally Lorentz invariant (Kibble type) state characterised, in accordance with the Goto-Nambu limit formula (1.1), by $U=T=m^{2}$, the surface energy density $U$ increases without limit as the tension $T$ decreases towards zero, while the temperature $\Theta$ also increases monotonically as $T$ decreases, though not without limit but only towards a finite maximum (Hagedorn type) saturation value $\Theta_{\max }$ that is of the same order of magnitude as the relevant cosmological symmetry breaking phase transition temperature at which the string formation occurred in the first place: it can be seen from (3.6) that explicitly, in terms of the relevant (Higgs) mass scale $m$, this maximum saturation temperature will be given by

$$
\Theta_{\max }=\sqrt{\frac{3}{2 \pi}} m,
$$


It is to be expected that the cosmic strings should originally have been formed with a temperature of effectively the same order as the Higgs mass scale characterising the symmetry breaking phase transition involved, i.e. with $\Theta \approx \Theta_{\max }$, but that they would at first have been strongly coupled to their environment and would therefore have cooled rapidly as the universe expanded so that by the time they emerged as effectively free systems governed without allowance for external drag corrections just by the Goto-Nambu action (1.2) in so far as small scale behaviour is concerned and by the standard "hot string" Lagrangian (3.1) at the level of larger scale averaged behaviour, the temperature would have been brought down to a very much lower value, $\Theta \ll \Theta_{\max }$. However although this implies that the in the short run the mechanical effects of the thermal current would be relatively small, it is by no means obvious that they would not become important in the long run provided the heat loss by thermal radiation is sufficiently small for the entropy current to be sufficiently well conserved over timescales long enough for a loop to have undergone significant overall contraction due to long wavelength gravitational radiation, since under such circumstances the current would ultimately become more densely concentrated with the consequence that the string would tend to heat up again. A quantitative analysis of the likely efficiency of this potentially important reheating effect is therefore an interesting subject for future work. 


\section{The need for composite "warm" cosmic string models.}

It is apparent that there is a sense in which the "hot" string model characterised by (3.1) - in which the only current is of entirely thermal origin - represents an opposite extreme from the "cold" limit represented by the Witten type "superconducting" (or in stricter terminology "superfluid") models characterised by a Lagrangian of the form (2.3) - in which there is no allowance for any thermal contribution at all. Models of the latter type are interpretable as the 1-dimensional analogues of an ordinary 3-dimensional superfluid of the simple single constituent type that is exemplified by ordinary liquid helium-4 in the limit of strictly zero temperature. It is however well known that when one needs to take account of the effects of finite temperature in liquid helium-4 one has to use a model of the two constituent type whose prototype was first set up by Landau. The essential feature of Landau's "warm" superfluid model ${ }^{[29]}$ is the coexistence of two independent (but of course strongly coupled) currents representing the independent degrees of freedom of a "cold" constituent, corresponding to the boson condensate, on one hand, and of a "hot" (or in the traditional technical jargon "normal") constituent, corresponding to the entropy current (which at low temperatures can be accounted for mainly in terms of "phonons"). In the context of cosmic strings, the analogue of the "cold" part is the Witten type condensate discussed in Section 2, while the analogue of the "hot" part is the thermal excitation current discussed in section 3. Our purpose here is to describe the way to allow for the presence of both kinds of current together in a "warm" superfluid cosmic string model that will be the 1 dimensional analogue of the ordinary 3-dimensional Landau model.

Before proceeding, it is to be remarked that the simple explicit formulae presented in the previous section allow for the macroscopic thermal description only for what might be described as "snake" modes but not for other more complicated possibilities (of which the most obvious are what might be described "sausage" modes) whose inclusion in a more accurate warm string model would presumably require numerical not just analytic calculations. When we start not from an underlying smallscale string that is not of the Goto Nambu type characterised by (1.2) that was considered in section 3 but of the "superconducting" type characterised by (2.3), the situation becomes even more complicated, because an explicit analytic treatment is not available even for the unperturbed equation of state, and hence a fortiori not for the thermal treatment of even the simplest "snake" type perturbation modes. Such a state of relative ignorance need not however discourage us from developing a formal framework for the qualitative analysis of variables whose explicit quantitative values one may hope to obtain later from 
detailed numerical computations, since this state of affairs is closely analogous to that which pertains to the familiar laboratory phenomenon of superfluidity in liquid helium - the only essential difference being that in the laboratory case results that are beyond the scope of present day numerical analysis may already be empirically accessible to some extent by experimental methods.

In the laboratory case a considerable body of experience encourages faith in the use, as a first approximation, of the standard Landau theory ${ }^{[29]}$ whose natural relativistically covariant generalisation of this theory has recently been shown ${ }^{[30]}$ to have a variational formulation in terms of a Lagrangian of the form

$$
L=L\left(\mu_{\rho}, s^{\rho}\right)
$$

that is specified as a scalar function of field variables that are taken to be a gradient $\mu_{\rho}$ constructed, as in (2.1), from the scalar phase $\varphi$ of an underlying quantum condensate (as in the single current string model governed by (2.3)) and an entropy current vector $s^{\rho}$ (as in the hot cosmic string model governed by (3.1)) whose variation is not considered to be free but subject to a constraint of convective type ${ }^{[31]}$. Of course if very high accuracy were required such a strictly conservative treatment would not be quite sufficient and would need to be modified to allow for effects such as viscosity of the entropy flux, but its sufficiency for many purposes, including the description of first and second sound perturbation modes, encourages its translation to the highly analogous (theoretically rather simpler though experimentally less accessible) context of cosmic strings for which the zero temperature limit, $s^{\rho}=0$ and the non "superconducting" limit $\mu_{\rho}=0$ are already familiar as the special cases given respectively by (2.3) and (3.1).

The way to do this is described in the following sections, in which it will be shown how to set up a class of "warm superfluid" $p$-brane models that includes the relativistic version of the ordinary Landau two constituent superfluid model in the case $p=3$ and that includes the "warm" conducting string model in which we are particularly interested as the case $p=1$. Since this model itself includes the original Witten type string model as a zero temperature "cold" limit, it can be seen that the fashionable use of the term "superconducting" is rather an exageration for describing this particular case since it corresponds in this categorisation to the simplist kind of superfluid as exemplified by zero temperature helium and not to a proper superconductor of the kind exemplified by many low temperature metallic media. (Such genuinely superconducting behaviour is indeed likely to be exhibited by cosmic strings of the more complicated type that arise from more realistic theories such as that of Peter ${ }^{[3]}$, but a simple Witten type model of 
the form characterised by (2.3) has too few degrees of freedom for superconductivity in the strict sense to be able to occur, and would therfore be more appropriately described as a "charged superfluid" string so as to leave the term "superconducting" string avilable for distinguishing the more complicated cases that remain as an interesting subject for future investigation).

\section{Entropy flux in a conservative $p$-brane model.}

In order to set up a category of models that manifestly includes both the relativistic version of the standard Landau two-constituent superfluid theory and also the warm "superconducting" cosmic string model in which we are most particularly interested as a special cases, we shall start by considering a $p$-brane of arbitrary dimension, using the standard convention ${ }^{[32]}$ that $p$ here refers to the dimension of a spacial section through the structure under consideration, which means that the dimension of its spacetime world sheet will be $p+1$. Thus the case of an ordinary superfluid will be specified by $p=3$, and the string case in which we are primarily interested here will be specified by $p=1$, while the intermediate case $p=2$ corresponds, at least in a 4 dimensional spacetime background, to the hypersurface supported case of a membrane. For the purpose of the present section the background spacetime dimension $n$ will not be restricted, so that hypothetical higher dimensional cases are also implicitly included. The position of the brane worldsheet relative to to local background spacetime coordinates $x^{\mu},(\mu=0,1, \ldots n-1)$ (where in the usual applications $\left.n=4\right)$ will therefore be given by a mapping of the form

$$
\sigma^{i} \mapsto x^{\mu}(\sigma)
$$

where the $\sigma^{i}, i=0,1, \ldots, p$, are internal coordinates on the $p$-brane world sheet. With respect to such a reference system the scalar field $\varphi$ (representing the superfluid phase angle) on which the models set up below will depend is simply given by another such internal coordinate mapping,

$$
\sigma^{i} \mapsto \varphi(\sigma)
$$

The essential feature of a "warm" as opposed to "cold" material medium or more general $p$ brane model is a current of entropy which will be supposed to be conserved in the not dissipative case to which the present discussion will be restricted (as it must be if a variational treatment is to be obtained). Such a current can be represented mathematically by a $p$-dimensional manifold $\mathcal{X}$ say with a corresponding $p$-surface measure $d S$ and local coordinates $X^{\mathrm{A}}$ say, $(A=1, \ldots, p)$, that is obtained as 
the image of a projection expressible in a form analogous to (5.1) and (5.2) as

$$
\sigma^{i} \mapsto X^{\mathrm{A}}(\sigma)
$$

With respect to the comoving entropy current coordinates $X^{\mathrm{A}}$ the entropy measure will be have an expression of the standard form

$$
d S=(p !)^{-1} S_{\mathrm{A}_{1} \ldots \mathrm{A}_{\mathrm{p}}} d X^{\mathrm{A}_{1}} \ldots d X^{\mathrm{A}_{\mathrm{p}}}
$$

where the $S_{\mathrm{A}_{1} \ldots \mathrm{A}_{\mathrm{p}}}$ are antisymmetric tensorial compenents that depend only on the comoving coordinates $X^{\mathrm{A}}$ (and that can easily be made uniform and normalised so as to have values \pm 1 or 0 by suitably adjusting these comoving coordinates) so that they will determine a corresponding $(p+1)$ surface current $p$-form that will have components

$$
S_{i_{1} \ldots i_{p}}=S_{\mathrm{A}_{1} \ldots \mathrm{A}_{\mathrm{p}}} X_{, i_{1}}^{\mathrm{A}_{1}} \ldots X_{, i_{p}}^{\mathrm{A}_{\mathrm{p}}}
$$

where a comma is used to indicate partial derivation with repect to the coordinates whose indices are indicated, and that will automatically be closed in the sense of having vanishing exterior derivative, i.e.

$$
(p+1) S_{\left[i_{1} \ldots i_{p}, i_{0}\right]}=0
$$

using square brackets to denote index antisymmetrisation. Since the world sheet will have its own geometric measure tensor, with components $\mathcal{E}_{i_{0} i_{1} \ldots i_{p}}$ say, that is determined (modulo a choice of sign expressing the orientation) in the usual way by the square root of the modulus of the determinent $|\eta|$ of the internal metric $\eta_{i j}$, where the latter is induced via (5.1) by the background metric $g_{\mu \nu}$ according to the usual pullback formula

$$
\eta_{i j}=g_{\mu \nu} x_{, i}^{\mu} x_{, j}^{\mu}
$$

it follows, raising internal indices by contraction with the inverse $h^{i j}$ of the internal metric tensor $h_{i j}$, that the entropy current $p$-form will have as its dual a corresponding entropy current vector

$$
s^{i}=(p !)^{-1} \mathcal{E}^{i j_{1} \ldots j_{p}} S_{j_{1} \ldots j_{p}}
$$

that will automatically satisfy the corresponding surface conservation law

$$
\left(\|\eta\|^{1 / 2} s^{i}\right)_{, i}=0
$$


The introduction of the fundamental tensor $\eta^{\mu \nu}$ as constructed ${ }^{[15][18]}$ from the inverse $h^{i j}$ of the internal metric according to the defining specification

$$
\eta^{\mu \nu}=\eta^{i j} x_{, i}^{\mu} x_{, j}^{\nu}
$$

enables us to complete the construction of the surface projected gradient operator $\widetilde{\nabla}_{\nu}$ as defined by (2.2) and hence to rewrite the conservation identity (5.9) in terms of the the the corresponding background current vector

$$
s^{\mu}=s^{i} x_{, i}^{\mu}
$$

in background tensorial notation as

$$
\widetilde{\nabla}_{\mu} s^{\mu}=0
$$

\section{Lagrangian displacement analysis in a brane model.}

The reason for going to the trouble of formally deriving the conservation law (5.9) as a consequence of the more fundamental relations (5.3), (5.4), and (5.5), rather than simply postulating postulating (5.9), or its tensorial version (5.12), directly as an axiom in its own right is that the action principle that we need is based on the free variation of the mappings (5.1), (5.2) and (5.3). This means that unlike the scalar field $\varphi$, the entropy current vector $s^{\mu}$ will not have the status of a independent field variable, its place being effectively taken by the comoving coordinate fields $X^{\mathrm{A}}$. However since the latter are not physically well defined, being subject to a very high degree of gauge dependence in so much as they are replaceable by arbitrary functions of themselves, it is desirable to introduce machinery whereby they can be eliminated as soon as possible in favour of more physically meaningful quantities such as $s^{\mu}$ itself. All we have to do to achieve this is to take over into brane theory the device whose use in the more familiar context of ordinary fluid theory is associated with the name of Lagrange, i.e. to work with a reference system that is itself comoving with respect to the variations to be considered. Thus we can arrange that the only change in $s^{i}$ is the part due to the variation of the measure, which just leaves

$$
\delta_{\mathrm{L}} s^{i}=-\frac{1}{2} s^{i} \eta^{j k} \delta_{\mathrm{L}} \eta_{j k}
$$

simply by choosing internal coordinates $\sigma^{i}$ for the perturbed system with respect to which the variations of the entropy space coordinates reduce to zero, i.e. for which the mapping (5.3) is preserved so that

$$
\delta_{\mathrm{L}} X^{\mathrm{A}}(\sigma)=0
$$


where $\delta_{\mathrm{L}}$ denotes the corresponding Lagrangian differential operator associated with the infinitesimal change under consideration (whereas if (6.2) were not imposed, the right hand side of (6.1) would have to be supplemented by a lot of terms involving not only the variations $\delta_{\mathrm{L}} X^{\mathrm{A}}$ themselves but also their derivatives $\delta_{\mathrm{L}} X_{, i}^{\mathrm{A}}$ ).

Since there are only $p$ independent comoving coordinates $X^{\mathrm{A}}$, while the $p$-brane world sheet is itself $(p+1)$ dimensional, it will be generically possible while we are about it, consistently with (6.2), to use the remaining freedom of internal coordinate adjustment to pin down the Lagrangian reference system completely by taking it to be comoving with the phase field as well, i.e. arranging to preserve the mapping (5.2) so as to have

$$
\delta_{\mathrm{L}} \varphi(\sigma)=0
$$

as well.

This still leaves us free to make what, for $p<n-1$ is the most important simplification of all, which is to take the background coordinates to be comoving with the $p$-brane world sheet itself, i.e. to freeze not just the entropy current mapping (5.3) as is done by (6.2) and the phase mapping (5.2) as is done by (6.3) but also to freeze the basis brane imbedding mapping (5.1) by arranging to have

$$
\delta_{\mathrm{L}} x^{\mu}(\sigma)=0
$$

with the corollary that the corresponding variation of the $(p+1)$ surface measure density will be given by

$$
\|\eta\|^{-\frac{1}{2}} \delta_{\mathrm{L}}\|\eta\|^{\frac{1}{2}}=\frac{1}{2} \eta^{i j} \delta_{\mathrm{L}} h_{i j}=\frac{1}{2} \eta^{\mu \nu} \delta_{\mathrm{L}} g_{\mu \nu}
$$

while that of the (rank $p+1)$ tangential projection tensor $\eta^{\mu}{ }_{\nu}$ will be given in terms of its (rank $n-p-1$ ) complement, the surface orthogonal projection tensor $\perp_{\nu}^{\mu}$ by

$$
\delta_{\mathrm{L}} \eta_{\sigma}^{\rho}=\eta^{\rho \mu} \perp_{\sigma}^{\nu} \delta_{\mathrm{L}} g_{\mu \nu}
$$

where the latter is got in its fully contravariant version $\perp^{\mu \nu}$ directly from the fundamental tensor $\eta^{\mu \nu}$ as defined by (5.10) by the defining relation

$$
\perp^{\mu \nu}=g^{\mu \nu}-\eta^{\mu \nu} .
$$

The use of such a fully Lagrangian system can be seen from (5.7) and (5.10) to have the advantage of making (6.1) immediately translatable into background tensorial form as

$$
\delta_{\mathrm{L}} s^{\rho}=-\frac{1}{2} s^{\rho} \eta^{\mu \nu} \delta_{\mathrm{L}} g_{\mu \nu}
$$


while for the momentum-energy covector $\mu_{\mu}$ constructed according to (2.1) as the gradient of the phase scalar $\varphi$ one obtains the corresponding relation

$$
\delta_{\mathrm{L}} \mu_{\rho}=\mu^{\nu} \perp_{\rho}^{\mu} \delta_{\mathrm{L}} g_{\mu \nu}
$$

\section{Semi Eulerian "surface sliding" displacement analysis in a brane model.}

Valuable though it is, the simplification (6.4) is seldom achieved without some cost in practice, since the choice of the background coordinates $x^{\mu}$ is usually not quite so arbitrary as that of the comoving current coordinates $X^{\mathrm{A}}$, particularly in cases when gravity is only involved weakly or not at all. It is usually the case that some criterion, such as flatness when gravity is absent, determines a corresponding "fixed" reference system which it would be traditional to refer to as "Eulerian", with corresponding local coordinates coordinates $z^{\mu}$ say that could be used to fix the choice of the $x^{\mu}$ in the unperturbed configuration, but which would be related to them by a nontrivial infinitesimal displacement mapping determined by a corresponding displacement vector field $\delta^{\mu}$ in the form

$$
x^{\mu} \mapsto z^{\mu}(x)=x^{\mu}+\delta^{\mu}(x)
$$

There is however no difficulty in translating from the Eulerian differential $\delta_{\mathrm{E}}$ say measuring the change of any background field quantity with respect to such a reference system - to the corresponding Lagrangian differential as denoted by $\delta_{\mathrm{L}}$, the difference being given ${ }^{[33][34]}$ simply by the Lie derivative $\overrightarrow{\delta \mathcal{E}}$ with respect to the relative displacement vector field $\delta^{\mu}$ determined by (7.1), i.e. one has

$$
\delta_{\mathrm{L}}=\delta_{\mathrm{E}}+\overrightarrow{\delta \mathcal{E}}
$$

However although an Eulerian reference system may be indispensible for the description of background spacetime fields, such as the metric itself (particularly in cases where the background spacetime is fixed to be flat so that one gets the convenient simplification $\delta_{\mathrm{E}} g_{\mu \nu}=0$ ), on the other hand an Eulerian description is useless for fields (such as $\varphi$ and $s^{\mu}$ in our present case) whose support is confined to a lower dimensional brane worldsheet. What can however be usefully done when worldsheet displacements are involved is to introduce an intermediate "surface sliding" reference system obtained by a displacement of the form

$$
x^{\mu} \mapsto y^{\mu}(x)=x^{\mu}+\zeta^{\mu}(x)
$$


where $\zeta^{\mu}$ is restricted to be tangential to the worldsheet, i.e.

$$
\perp^{\mu}{ }_{\nu} \zeta^{\nu}=0
$$

so that it will be obtainable, by applying the brane imbedding mapping (5.1) to a corresponding internal worldsheet vector with components $\zeta^{i}$, in the form

$$
\zeta^{\mu}=\zeta^{i} x_{, i}^{\mu}
$$

which means that there will be a corresponding infinitesimal internal coordinate transformation

$$
\sigma^{i} \mapsto \sigma^{i}+\zeta^{i}
$$

whose application in conjunction with (7.3) will preserve the form of the basic imbedding mapping (5.1), thereby providing us with a new semi-Lagrangian, semi-Eulerian, reference system (one that is Lagrangian from the point of view of the background coordinates $x^{\mu}$ but that is Eulerian from the point of view of the internal coordinates $\sigma^{i}$ ) which will characterise a corresponding "surface sliding" differential, $\delta_{\mathrm{S}}$ say, that will satisfy a differential imbedding conservation law

$$
\delta_{\mathrm{S}} x^{\mu}(\sigma)=0
$$

of the same form as its fully Lagrangian analogue (6.4), with corollaries analogous to (6.5) and (6.6), i.e.

$$
\|\eta\|^{-\frac{1}{2}} \delta_{\mathrm{S}}\|\eta\|^{\frac{1}{2}}=\frac{1}{2} h^{i j} \delta_{\mathrm{S}} \eta_{i j}=\frac{1}{2} \eta^{\mu \nu} \delta_{\mathrm{L}} g_{\mu \nu}
$$

and

$$
\delta_{\mathrm{S}} \eta_{\sigma}^{\rho}=\eta^{\rho \mu} \perp_{\sigma}^{\nu} \delta_{\mathrm{S}} g_{\mu \nu}
$$

but which, unlike its fully Lagrangian anologue, will not be subject to purely internal conservation conditions analogous to (6.2) and (6.3).

For our present purposes, the way of specifying such a semi-Eulerian refence system that is most convenient is to postulate that (instead of obeying the analogues of (6.2) and (6.3)) it should be characterised by the requirement that the further non tangential displacement

$$
y^{\mu} \mapsto z^{\mu}=y^{\mu}+\xi^{\mu}
$$

say, with $\xi^{\mu}$ specified by a decomposition of the form

$$
\delta^{\mu}=\zeta^{\mu}+\xi^{\mu}
$$


that must be superimposed on (7.3) in order to get back to (7.1), should satisfy the orthogonality condition that is the obvious complement of (7.4), i.e.

$$
\eta_{\nu}^{\mu} \xi^{\nu}=0
$$

Having specified the decomposition (7.11) in this way, we can think of the translation (7.2) as the result of successively performing two subsidiary translations, namely a worldsheet orthogonal displacement whose effect is expressible by

$$
\delta_{\mathrm{S}}=\delta_{\mathrm{E}}+\overrightarrow{\xi E}
$$

and a worldsheet tangential displacement whose effect is expressible by

$$
\delta_{\mathrm{L}}=\delta_{\mathrm{S}}+\overrightarrow{\zeta E}
$$

of which the former is applicable only to background spacetime fields such as the metric $g_{\mu \nu}$ itself, whereas the latter, (7.14) is equally well applicable also to fields (such as $\varphi$ and $s^{\mu}$ ) whose support is confined to the brane worldsheet.

The upshot of all this work is that the elimination of the variations of the $p+1$ independent field components consisting of the $X^{\mathrm{A}}$ and of $\varphi$ by the imposition of the Lagrangian displacement conditions (6.2) and (6.3), and the further elimination by (6.4) of the $n-p-1$ degrees of freedom of adjustment of the location of the $(p+1)$ dimensional world sheet in directions orthogonal to itself is compensated by the introduction of the $n$ new degrees of freedom of adjustment of the components of the relative displacement vector $\delta^{\mu}$, the components $\zeta^{\mu}$ representing the $p$ degrees of freedom of the $X^{\mathrm{A}}$ together with the single degree of freedom of $\varphi$, while the comonents $\xi^{\mu}$ correspond to the remaining $n-p-1$ degrees of freedom of adjustment of the brane locus. This means that for the purposes of the action principle the independent infinitesimal variations of the mappings (5.1), (5.2), and (5.3) are effectively and conveniently replaced, in a Lagrangian reference system as characterised by (6.2), (6.3), (6.4), by the independent variation of the components just of the single infinitesimal displacement vector $\delta^{\mu}$ or equivalently, in a semi Eulerian system as characterised by (7.7), by the variation, subject the constraints (7.4) and (7.12), of the separate vector fields $\zeta^{\mu}$ and $\xi^{\mu}$. 


\section{The action for a "warm" superfluid $p$-brane model.}

The action that we shall use has the form of a $p$-brane world sheet integral

$$
\mathcal{I}=\int \mathcal{L} d^{p+1} \sigma
$$

in which the action density $\mathcal{L}$ has the form

$$
\mathcal{L}=\|\eta\|^{\frac{1}{2}} L
$$

where $|\eta|$ is the determinant of the internal $(p+1)$ surface metric $\eta_{i j}$ as given by (5.7) and $L$ itself is a Lagrangian scalar of the form (4.1).

In evaluating the variation of this integral it will be most instrictive to use the intermediate semi-Eulerian reference system, in terms of which we shall have

$$
\delta_{\mathrm{S}} \mathcal{I}=\int\left(\delta_{\mathrm{S}} \mathcal{L}\right) d^{p+1} \sigma
$$

with

$$
\delta_{\mathrm{S}} \mathcal{L}=\|\eta\|^{\frac{1}{2}} \diamond_{\mathrm{S}} L
$$

where $\nabla_{\mathrm{S}}$ is a modified pseudo differential operator given by

$$
\diamond_{\mathrm{S}}=\delta_{\mathrm{S}}+\frac{1}{2}\left(\eta^{\mu \nu} \delta_{\mathrm{S}} g_{\mu \nu}\right)
$$

whose introduction in place of $\delta_{\mathrm{S}}$ allows us to work with exclusively tensorial quantities by including an adjustment term allowing for the variation $(7.8)$ of the $(p+1)$ surface measure density factor $\|\eta\|^{\frac{1}{2}}$.

For the evaluation of the variation in a compact region of the integral as a whole it would of course make no difference whether we use a fully Lagrangian or a semi-Eulerian reference system, i.e. we have

$$
\delta_{\mathrm{L}} \mathcal{I}=\delta_{\mathrm{S}} \mathcal{I}
$$

since the difference in the corresponding integrands will just have the form of a pure $(p+1)$ surface divergence, i.e.

$$
\diamond_{\mathrm{L}} L=\diamond_{\mathrm{S}} L+\widetilde{\nabla}_{\nu}\left(\zeta^{\nu} L\right)
$$

where $\zeta^{\nu}$ is the tangential displacement vector introduced in the previous section.

Since $L$ is postulated to be just a covariant scalar function of the entropy current vector $s^{\nu}$ as constructed in section 5, and of the momentum-energy covector 
$\mu_{\nu}$ that is constructed in accordance with (2.1) as the gradient of the phase scalar $\varphi$, it follows that its semi Eulerian differential will be expressible in terms of a thermal momentum-energy covector $\Theta_{\nu}$ that is the dual of $s^{\mu}$ and of a condensate current vector $\nu^{\mu}$ that is the dual of $\mu_{\nu}$ in the form

$$
\delta_{\mathrm{S}} L=\Theta_{\nu} \delta_{\mathrm{S}} s^{\nu}-\nu^{\nu} \delta_{\mathrm{S}} \mu_{\nu}+\frac{1}{2} \eta^{\mu \rho}\left(\Theta_{\rho} s^{\nu}+\mu_{\rho} \nu^{\nu}\right) \delta_{\mathrm{S}} g_{\mu \rho}
$$

where the form of the coefficient of the metric variation in the final term is easily derivable (as a Noether identity ${ }^{[31]}$ ) from the covariance condition, and where, in view of the tangentiality constraints

$$
s^{\rho} \perp_{\rho}^{\mu}=0, \quad \mu^{\nu} \perp_{\rho}^{\nu}=0
$$

to which $s^{\rho}$ and $\mu_{\rho}$ are subject by construction, it is to be understood that the specification of the partial derivatives is resolved by the imposition of corresponding tangentiality conditions

$$
\Theta^{\nu} \perp_{\rho}^{\nu}=0, \quad \nu^{\rho} \perp_{\rho}^{\mu}=0 .
$$

It is to be remarked that just as the construction of the current $s^{\rho}$ automatically ensures that it will satisfy the conservation law (5.8), so analogously the construction of the energy-momentum covector $\mu_{\rho}$ as a tangential gradient by (2.1) automatically ensures that it will satisfy a corresponding conservation law as a consequence of the commutator identity

$$
\widetilde{\nabla}_{[\mu} \widetilde{\nabla}_{\nu]} \varphi=K_{[\mu \nu]}^{\rho}{ }^{\rho} \widetilde{\nabla}_{\rho} \varphi
$$

which must be satisfied by any scalar field $\varphi$, where $K_{\mu}{ }^{\rho}{ }_{\nu}$ is the second fundamental tensor (which determines both the inner and the outer curvature ${ }^{[21][36][37]}$ of the world sheet) as defined ${ }^{[15][18]}$ in terms of the first fundamental tensor $\eta_{\mu}{ }^{\nu}$ by the construction

$$
K_{\mu \nu}{ }^{\rho}=\eta_{\mu}^{\sigma} \widetilde{\nabla}_{\nu} \eta_{\sigma}^{\rho}
$$

which not only ensures the obvious tangentiality and orthogonamity properties

$$
\perp_{\sigma}^{\mu} K_{\sigma \nu}{ }^{\rho}=0=K_{\mu \nu}{ }^{\sigma} \eta_{\sigma}{ }^{\rho}
$$

but also (as a surface integrability condition) the non-trivial Weingarten identity

$$
K_{[\mu \nu]}^{\rho}=0 .
$$

One thus obtains a corresponding identity of the form

$$
\widetilde{\nabla}_{[\mu} \mu_{\nu]}=K_{[\mu}^{\rho}{ }^{\rho}{ }_{\nu]} \mu_{\rho}
$$

as the integrability condition for $\mu_{\rho}$ to have the form (2.1). 


\section{Dynamics of a "warm" superfluid $p$-brane model.}

To deal with the internal part of the variation what now has to be done is to apply the translation relation (7.14) to the Lagrangian formulae (6.8) and (6.9) for $s^{\rho}$ and $\mu_{\rho}$ we obtain the corresponding semi Eulerian variations needed for working out (8.8) in the form

$$
\delta_{\mathrm{S}} s^{\rho}=-\frac{1}{2} s^{\rho} \eta^{\mu \nu} \delta_{\mathrm{S}} g_{\mu \nu}+s^{\rho} \widetilde{\nabla}_{\nu} \zeta^{\nu}-\zeta^{\nu} \widetilde{\nabla}_{\nu} s^{\rho}+s^{\nu} \widetilde{\nabla}_{\nu} \zeta^{\rho}
$$

and

$$
\delta_{\mathrm{S}} \mu_{\rho}=\mu^{\nu} \perp_{\rho}^{\mu}\left(\delta_{\mathrm{S}} g_{\mu \nu}+\widetilde{\nabla}_{\nu} \zeta_{\mu}\right)-\zeta^{\nu} \widetilde{\nabla}_{\nu} \mu_{\rho}-\mu_{\nu} \widetilde{\nabla}_{\rho} \zeta^{\nu}
$$

from which one immediately obtains in variational integrand in (8.4) is the in the form

$$
\diamond_{\mathrm{S}} L=\frac{1}{2} \widetilde{T}^{\mu \nu} \delta_{\mathrm{S}} g_{\mu \nu}+\Theta_{\rho}\left(s^{\nu} \widetilde{\nabla}_{\nu} \zeta^{\rho}-\zeta^{\nu} \widetilde{\nabla}_{\nu} s^{\rho}\right)+\nu^{\rho}\left(\mu_{\nu} \widetilde{\nabla}_{\rho} \zeta^{\nu}+\zeta^{\nu} \widetilde{\nabla}_{\nu} \mu_{\rho}\right)
$$

where the the coefficient $\widetilde{T}^{\mu \nu}$ of the metric variation, which is of course to be interpreted as the surface stress momentum energy tensor, is given by

$$
\widetilde{T}_{\nu}^{\mu}=s^{\mu} \Theta_{\nu}+\nu^{\mu} \mu_{\nu}+\Psi \eta_{\nu}^{\mu}
$$

in which the scalar potential $\Psi$, which is interpretable as a generalised pressure function, has the form

$$
\Psi=L-s^{\nu} \Theta_{\nu}
$$

To deal with the external part of the variation, i.e. the part concerned with the displacement of the world sheet itself, it remains to apply the translation relation (7.13) to the metric, which gives

$$
\delta_{\mathrm{S}} g_{\mu \nu}=\delta_{\mathrm{E}} g_{\mu \nu}+2 \nabla_{(\mu} \xi_{\nu)}
$$

In order to proceed with the application of the variation principle without having to worry about ultraviolet divergence and renormalisation effects, we now make the postulate that the background spacetime remains fixed. This means discounting backreaction effects due to emmission of gravitational radiation, which will be an extremely good approximation not only for "lightweight" string models characterised by $G m^{2} \approx 10^{-32}$ but even the "heavyweight" G.U.T. string models characterised by $G m^{2} \approx 10^{-6}$ which is still sufficiently small for it to be entirely satisfactory to ignore gravitational radiation in the treatment of the short run dynamical behaviour, and to take account of the 
backreaction phenomenon only as a mechanism of slow "secular" energy loss ${ }^{[33][34][35]}$ that is of physical significance only as a statistically accumulative effet in the very long run. This understanding allows us to take the Eulerian part of the metric perturbation here simply to be zero,

$$
\delta_{\mathrm{E}} g_{\mu \nu}=0
$$

thereby completing the reduction of the problem to the required form in which the only independent variations are those of the tangential and orthogonal displacement vectors $\zeta^{\nu}$ and $\xi^{\nu}$.

We now come to the final step in the application of the variation principle which is traditionally performed by combining the derivatives of the independent variables into divergences that can be integrated out using Green's theorem leaving only undifferentiated terms whose coefficients are the Eulerian derivatives that must be set to zero as the dynamical equation. As far as the derivatives tangential displacement variable $\zeta$ is concerned we shall now go ahead in this traditional way using the symmetry condition

$$
\nu^{\mu} \zeta^{\nu} \widetilde{\nabla}_{[\mu} \mu_{\nu]}=0
$$

that is obtained from (8.15) using (8.13), but to deal with the derivatives of the orthogonal displacement variable $\xi^{\nu}$ we shall use a trick of a less traditional but even more effective kind, differentiating the orthogonality condition (7.12) itself, which gives the relation

$$
\eta_{\rho}^{\nu} \widetilde{\nabla}_{\mu} \xi^{\rho}=-K_{\mu \rho}^{\nu} \xi^{\rho}
$$

which allows local elimination (without any integration or use of Green's theorem) of the derivatives of $\xi^{\mu}$. This strategy converts the variational integrand in (8.4) to the final form

$$
\diamond_{S} L=\widetilde{T}^{\mu \nu} K_{\mu \nu \rho} \xi^{\rho}-\zeta^{\rho}\left(2 s^{\mu} \widetilde{\nabla}_{[\mu} \Theta_{\rho]}+\mu_{\rho} \widetilde{\nabla}_{\mu} \nu^{\mu}\right)+\nabla_{\mu}\left\{2 \zeta^{[\rho} s^{\mu]} \Theta_{\rho}+\zeta^{\rho} \mu_{\rho} \nu^{\mu}\right\}
$$

It is now immediately apparent that the requirement that the the corresponding integral variation (8.4) should vanish for an arbitrary orthogonal displacement $\xi^{\rho}$ of the world sheet entails the external force balance condition

$$
\widetilde{T}^{\mu \nu} K_{\mu \nu \rho}=0
$$

which is of a form that is applicable ${ }^{[18][36]}$ to any isolated unpolarized brane model, the specific form of the stress momentum energy tensor (as given by (9.4) in this particular case) being all that distinguishes one kind of model from another. 
Since the last term in (9.6) has the form of a worldsheet divergence that integrates out, the requirement that the integral variation (8.4) should also vanish for an arbitrary tangential displacement $\zeta^{\nu}$ evidently entails that the requisite internal dynamical equations be given by

$$
\eta_{\nu}{ }^{\rho}\left(2 s^{\mu} \widetilde{\nabla}_{[\mu} \Theta_{\rho]}+\mu_{\rho} \widetilde{\nabla}_{\mu} \nu^{\mu}\right)=0 .
$$

By contracting this with $s^{\nu}$ it can be seen (subject to the assumption implicit throughout his work that $s^{\rho}$ a nd $\mu_{\rho}$ are both strictly timelike so that their product $s^{\nu} \mu_{\nu}$ will not vanish) that we are left with a condensate surface current conservation law of the form

$$
\widetilde{\nabla}_{\mu} \nu^{\mu}=0,
$$

which implies that the remaining content of (8.11) will reduce to the form

$$
\eta_{\nu}{ }^{\rho} s^{\mu} \widetilde{\nabla}_{[\mu} \Theta_{\rho]}=0
$$

which is the natural generalisation of the thermal momentum transport in the ordinary superfluid case ${ }^{[38]}$ to which it reduces in the case $p+1=n$ for which $\eta_{\nu}{ }^{\rho}$ is just the identity projector $g_{\nu}{ }^{\rho}$.

\section{Duality in the dynamics of warm string models.}

The outcome of the preceeding work was that he complete set of dynamical equations of motion of the warm $p$ brane model would be given by the external dynamical equation of motion (9.10), the internal dynamical equations (9.12) and (9.13), together with the defining relation (2.1) for $\mu_{\rho}$ in terms of $\varphi$, or equivalently the corresponding integrability condition (8.15) whose essential surface projected part is just the symmetry condition

$$
\eta_{\mu}{ }^{\rho} \eta_{\nu}{ }^{\sigma} \widetilde{\nabla}_{[\rho} \mu_{\sigma]}=0
$$

and finally the entropy conservation law (5.12) which is formally analogous to the dynamic current conservation law (9.12), but whose status in the present variational formulation is merely that of a kinematic identity.

Although the currents $s^{\rho}$ and $\nu^{\rho}$ have qualitatively very different roles in the variational formulation used here, which is the one that arised most naturally from the context in which the concept of such a "warm" brane model originated - it is nevertheless to be emphasized that there will be no such qualitative distinction within 
the physical "on shell" mechanical model that is ultimately obtained. It is manifestly apparent ${ }^{[39]}$ in the present relativistic version (and, though it was not so obvious in the notation that was traditionally used in the past, can also be demonstrated ${ }^{[40]}$ in the original Newtonian version) of the Landau superfluid model that there is a strong analogy between the roles of the entropy current $s^{\rho}$ and its dynamical conjugate, the thermal momentum energy covector $\Theta_{\rho}$ on one hand, and the roles of the condensate current $\nu^{\rho}$ and its dynamical conjugate, the condensate momentum energy covector $\mu_{\rho}$ on the other hand.

In the particular case of a string, with $p=1$, to which we shall from this point on again restrict our attention, the analogy that was discernible in the ordinary fluid case becomes perfect, since the fact that the string world sheet is only two dimensional means that the basic thermal dynamical equation (9.16) will in this case reduce simply to an irrotationality condition of the same form as the integrability condition (10.1) obeyed by $\mu_{\rho}$, i.e. we shall be left simply with

$$
\eta_{\mu}{ }^{\rho} \eta_{\nu}{ }^{\sigma} \widetilde{\nabla}_{[\rho} \Theta_{\sigma]}=0
$$

An immediate consequence of this is that just as $\mu_{\rho}$ is derivable globally from the condensate phase scalar $\varphi$, so also the thermal momentum covector will be at least locally derivable from a formally analogous thermal gauge potential, $\vartheta$ say, via an analogous relation of the form

$$
\Theta_{\rho}=\widetilde{\nabla}_{\rho} \vartheta .
$$

In addition to this perfect "chemical symmetry" between the roles of the two independent constituents, the "warm" string case is characterised by a "dual symmetry" of the kind whose existence was previously noticed ${ }^{[23]}$ in the "cold" case, but which is more interesting in the present case becase the discrete chemical and dual symmetries combine to generate a forefold group of permutations among two pairs of 4 algebraicly (though not dynamically) independent conserved currents. The currents involved are the ordinary entropy current $s^{\rho}$ and the condensate current $\nu^{\rho}$ that have already been introduced, together with their respective duals, which are a thermal momentum vector $\star \Theta^{\rho}$ and a condensate momentum vector $\star \nu^{\rho}$ defined in terms of the background pullback

$$
\mathcal{E}^{\mu \nu}=\mathcal{E}^{i j} x_{, i}^{\mu} x^{\nu}, j
$$

of the internal worldsheet alternating tensor (as used in (5.7)) by the relations

$$
\star \Theta^{\rho}=\mathcal{E}^{\rho \nu} \Theta_{\nu}, \quad \star \star \mu^{\rho}=\mathcal{E}^{\rho \nu} \mu_{\nu}
$$


which are of course invertible to give

$$
\Theta_{\rho}=\mathcal{E}_{\rho \nu} \star \Theta^{\nu}, \quad \mu_{\rho}=\mathcal{E}_{\rho \nu} \star \mu^{\nu}
$$

In terms of these dual variables the "closure" conditions (10.1) and (10.3) translate into ordinary surface current conservation laws, so that the complete set of internal equations of motion for the warm string model reduces to a set of 4 such conservation laws, consisting of two mtually dual pairs i.e. a thermal pair

$$
\widetilde{\nabla}_{\nu} \star \Theta^{\nu}=0=\widetilde{\nabla}_{\nu} s^{\nu}
$$

and its chemical analogue, the condensate pair

$$
\widetilde{\nabla}_{\nu} \star \mu^{\nu}=0=\widetilde{\nabla}_{\nu} \nu^{\nu}
$$

The surface stress momentum energy tensor appearing in the equation (9.13) for the external motion will be expressible in a form that is manifestly both chemically and dually symmetric as

$$
\widetilde{T}_{\sigma}^{\rho}=\frac{\Lambda}{\Lambda-\Psi}\left(s^{\rho} \Theta_{\sigma}+\nu^{\rho} \mu_{\sigma}\right)+\frac{\Psi}{\Psi-\Lambda}\left(\star \Theta^{\rho} \star s_{\sigma}+\star \mu^{\rho} \star \nu_{\sigma}\right)
$$

where the entropy and condensate current duals are given by

$$
\star s_{\rho}=\mathcal{E}_{\rho \sigma} s^{\sigma}, \quad \star \nu_{\rho}=\mathcal{E}_{\rho \sigma} \nu^{\sigma}
$$

while the dynamical conjugate $\Lambda$ of the pressure function $\Psi$, is given by

$$
\Lambda=L+n^{\rho} \mu_{\rho}
$$

so that by $(9.5)$ we have

$$
\Psi-\Lambda=-s^{\rho} \Theta_{\rho}-n^{\rho} \mu_{\rho}=\star \Theta^{\rho} \star s_{\rho}+\star \mu^{\rho} \star \nu_{\rho} .
$$

It is to be remarked that the (negative) scalar $\Lambda$ introduced in this way could be used as a Lagrangian for an alternative variational formulation of purely convective type ${ }^{[37]}$ in which the independent currents $s^{\nu}$ and $\nu^{\nu}$ are treated on the same footing. 


\section{Standard form of the stress momentum energy tensor.}

It is instructive to express the resutlts in terms of the standard the preferred rest frame specified by the timelike unit eigenvector $u^{\rho}$ of $\widetilde{T}_{\rho}^{\nu}$ which will have the form

$$
u^{\rho}=(\Theta s+\mu \nu)^{-1}\left(\Theta s^{\rho}+\mu \nu^{\rho}\right), \quad u^{\rho} u_{\rho}=-1
$$

and of the corresplonding spacelike unit eigenvector

$$
v^{\rho}=\mathcal{E}^{\rho \sigma} u_{\sigma}, \quad v^{\rho} v_{\rho}=1
$$

with the rest frame temperature $\Theta$ and the rest frame chemical potential $\mu$ defined by

$$
\Theta=-u^{\rho} \Theta_{\rho}, \quad \mu=-u^{\rho} \mu_{\rho},
$$

while the corresponding rest frame number densities are defined by

$$
s=-u_{\rho} s^{\rho}, \quad \nu=-u_{\rho} n u^{\rho} .
$$

In terms of this preferred fram we can express the surface stress energy momentum tensor in the standard form

$$
\widetilde{T}^{\rho \sigma}=U u^{\rho} u^{\sigma}-T v^{\rho} v^{\sigma}
$$

in which the eigenvalues $U$, the mass-energy per unit length, and $T$, and the string tension, are obtainable from the relations

$$
U+T=-\Lambda-\Psi, \quad U-T=\Xi
$$

where $\Xi$ is a positive scalar quantity $\Xi$ defined by

$$
\Xi^{2}=\left(s^{\rho} \Theta_{\rho}-n^{\rho} \mu_{\rho}\right)^{2}+4 s^{\rho} \mu_{\rho} \nu^{\sigma} \Theta_{\sigma},
$$

from which it can be seen that the energy per unit length will be expressible as

$$
U=\Theta s+\mu \nu-\Psi
$$

while the corresponding dual formula giving the string tension will be

$$
T=-\Lambda-\Theta s-\mu \nu
$$


(It is to be noted that in order for $T$ to be positive like $U$ it is necessary for $\Lambda$ to be negative.)

\section{Conserved numbers and equilibrium conditions.}

It follows from the the original current conservation laws (5.12) and (9.15) for $s^{\rho}$ and $\nu^{\rho}$ that their dual 1-forms as defined by (10.10) should satisfy irrotationality conditions analogous to (10.1) and (10.2) and should therefore be locally derivable from stream functions $S$ and $\psi$ say in the form

$$
\star s_{\rho}=\widetilde{\nabla}_{\rho} S, \quad \star \nu_{\rho}=\nabla_{\rho} \psi
$$

where $S$ will evidently be a measure of the entropy as originally introduced in section 5 , while $\psi$ will be analogues measure of the conserved flux associated with the condensate. In the case of a closed string loop, these will determine corresponding global circuit integrals

$$
[S]=\oint \star s_{\rho} d x^{\rho}=\oint S_{, i} d \sigma^{i}, \quad[\psi]=\oint \star \nu_{\rho} d x^{\rho}=\oint \psi_{, i} d \sigma^{i},
$$

that will be conserved in the strong sense of being unaffected by arbitrary continuous displacements of the circuit. Dually corresponding globally conserved quantities are of course also obtained from the original condensate phase scalar $\varphi$ as introdiced in (2.1) and its thermal analogue as introduced in (10.3), in the form

$$
[\varphi]=\oint \mu_{\rho} d x^{\rho}=\oint \varphi_{, i} d \sigma^{i}, \quad[\psi]=\oint \star \Theta_{\rho} d x^{\rho}=\oint \vartheta_{, i} d \sigma^{i}
$$

where $[\varphi]$ is interpretable as the total condensate phase winding angle while $[\vartheta]$ is analogously interpretable as a thermal winding angle. Thus whereas the loops of the simple "hot" model described in section 3 and of the opposite extreme case of the "cold" models described in section 2 are characterised by just two independent globally conserved quantities, namely $[S]$ and $[\vartheta]$ in the "hot" case, and $[\psi]$ and $[\varphi]$ in the cold case, in the case of the more elaborate "warm" models set up here a string loop is characterised by all four of these quantities independently.

It is the existence of such conserved quantities that makes it evident that (in contrast with the degenerate special case of Goto Nambu string loops) these various kinds of "hot" "warm" and "cold" string loops cannot just disappear by macroscopic radiation processes but, if left free from external perturbation, will instead presumably tend to settle down in the towards stationary equilibrium states that minimise the 
mass energy $\mathcal{M}$ say for the given values of the constants $[S],[\vartheta],[\psi],[\varphi]$. Of course in the very long run the assumptions on which these conservative "hot" "warm" and "cold" string models are based will cease to be exactly valid: entropy can gradually be lost by microscopic thermal radiation processes, and although topologically conserved in a classical description even the phase winding number $[\varphi]$ in the "cold" limit case can in principle decrease by quantum tunnelling. The latter effect has already been investigated to some extent ${ }^{[5]}$, what transpires being that the result is highly model dependent, leading to decay timescales in some cases so short as to be comparable with the dynamical timescales, in which case the classical string description breaks down completely, but in other cases, still within physically realistic parameter ranges, the tunnelling timescales are cosmologically long, leading, as noted in the introduction, to the possibility of a catastrophic mass excess for strings of the heavyweight type. It is evident that allowance for the thermal effect will tend to further enhance the long term survival capability of the string loops, how much so being a subject for future work.

The simplest configurations for the stationary ring states that will ultimately be approached so long as the conservative description used here remains valid will be of the circular rotating ring type that first considered in detail by Davis and Shellard ${ }^{[4][5][6]}$. A wider study for generic string models not only of circular equilibrium states $^{[18][19]}$ but also of more general - deformed - equilibrium states ${ }^{[20][21]}$ shows that the equilibrium of an isolated string loop in a flat background is characterised by a longitudinal running velocity $v$ (which in the circular case is the product of the radius $r$ and the angular velocity $\Omega$ say, i.e. $v=r \Omega$ ) whose magnitude must be the same as the extrinsic perturbation speed $c_{\mathrm{E}}$ as given by the formula in (3.4) which remains valid in the generic case (whereas the formula given for the longitudinal perturbation speed $c_{\mathrm{L}}$ ceases to be valid for the "warm" models considered here, which, as in the ordinary Landau superfluid, are characterised by not one but two distinct "first" and "second" longitudinal characteristic speeds). This means that we can take over from our previous analysis the conclusion that the longitudinal running speed in an equilibrium state will be given by

$$
v^{2}=\frac{T}{U}
$$

As in the simpler cases considered previously the intrinsic string state, which in this case depends on just the three independents scalars that can be constructed from $s^{\rho}$ and $\mu_{\rho}$, must be spacially as well as temporally uniform, since there will be no less than four independent Bernouilli type constants obtained by contracting the Killing vector $k^{\rho}$ say generating the stationary symmetry with the four independent conserved momentum 
1-forms, whose invariance with the stationary symmetry action is expressible, as a consequence of their internal irrotationality, by the uniformity conditions

$$
\widetilde{\nabla}_{\rho}\left(k^{\sigma} \Theta_{\sigma}\right)=\widetilde{\nabla}_{\rho}\left(k^{\sigma} \star s_{\sigma}\right)=\widetilde{\nabla}_{\rho}\left(k^{\sigma} \mu_{\sigma}\right)=\widetilde{\nabla}_{\rho}\left(k^{\sigma} \star \nu_{\sigma}\right)=0
$$

Thus again as in the simpler cases considerered previously, the total mass energy $\mathcal{M}$ will be expressible in terms of the circumerence

$$
\ell=\oint d \ell, \quad d \ell^{2}=\eta_{i j} d \sigma^{!} d \sigma^{j}
$$

(which in the circular case will be given by $\ell=2 \pi r$ ) and of the uniform values of the energy per unit length $U$ (in the comoving rest frame) and of the tension $T$ in the form

$$
\mathcal{M}=(U+T) \ell
$$

while the magnitude $\mathcal{J}$ say of the angular momentum will be given by

$$
(2 \pi \mathcal{J})^{2} \leq U T \ell^{4}
$$

with equality in the circular case for which the angular momentum takes the maximum value allowed by the four global constants $[S][\vartheta][\psi][\varphi]$ characterising the loop, in terms of which the right hand side of equation (12.8) will be given directly by

$$
U T \ell^{4}=([\vartheta][S]+[\varphi][\psi])^{2}
$$




\section{Referencses}

[1] N.K. Nielsen, P. Olesen, Nucl. Phys. B61 (1973) 45. 829 (1987).

[2] T.W.B. Kibble, J.Phys. A9 (1977) 1387.

[3] P. Peter, Phys. Rev. D46 (1992) 3322.

[4] R.L. Davis, E.P.S. Shellard, Phys. Lett B209 (1988) 485.

[5] R.L. Davis, Phys. Rev D38 (1988) 3722.

[6] R.L. Davis, E.P.S. Shellard, Nucl. Phys. B323 (1989) 209.

[7] B. Carter, inEarly Universe and Cosmic Structures (Xth Moriand Astrophysics Meeting) ed A. Blanchard et al (Editions Frontières, Gif-sur-Yvette, 1991) 273.

[8] B. Carter, Ann. N. Y. Acad. Sci. 647 (1991) 758.

[9] J. Ostriker, C. Thompson, E. Witten Phys. Lett. B180 (1986) 231.

[10] E. Copeland, M. Hindmarsh, N. Turok, Phys. Rev. Lett. 58, (1987) 1910.

[11] D. Haws, M. Hindmarsh, N. Turok, Phys. Lett. B209 (1988) 225.

[12] E. Copelans, D. Haws, M. Hindmarsh, N. Turok, Nucl. Phys. B306 (1988) 908.

[13] C. Hill, H. Hodges, M. Turner, Phys. Rev. D37 (1988) 263.

[14] A. Babul, T. Piran, D.N. Spergel, Phys. Lett. B202 (1988) 207.

[15] B. Carter, Phys. Lett. B228 (1989) 446.

[16]P. Peter, Phys. Rev. D46 (1992) 3335.

[17] P. Peter, Phys. Rev. D47 (1993) 3169.

[18] B. Carter, "Covariant Mechanics of Simple ans Conducting Strings and Membranes", in The Formation and Evolution of Cosmic Strings, ed G. Gibbons, S. Hawking, T. Vachaspati (Cambridge U.P., 1990) 213.

[19] B. Carter, Phys. Lett. B238 (1990) 166.

[20] B. Carter, V.P. Frolov, O. Heinrich, Class. and Quantum Grav. 8 (1991) 135.

[21] B. Carter, "Basic Brane Mechanics", in Relativistic Astrophysics and Gravitation (proc 10th Potsdam Seminar, Oct. 1991) ed. S. Gottloeber, J.P. Muecket \& V. Mueller, (World Scientific, Singapore, 1992) 300.

[22] E. Witten, Nucl. Phys. B249 (1985) 557.

[23] B. Carter, Phys. Lett. B224 (1989) 61.

[24] P. Fayet, Nucl. Phys. B347 (1990) 743. 
[25] N. Dowrick, N.A. McDougal, Phys. Rev. D38 (1988) 3699.

[26] B. Carter, Phys. Rev. D41 (1990) 3872.

[27] A. Vilenkin, Phys. Rev. D41 (1990) 3038.

[28] N.K. Nielsen, Nucl. Phys. B167 (1980) 249.

[29] T.W.B. Kibble, "Cosmic strings", in The Formation and Evolution of Cosmic Strings, ed G. Gibbons, S.W. Hawking, T. Vachaspati (Cambridge U.P., 1990) 3.

[30] L.D. Landau, E.M. Lifshitz, Course of Theoretical Physics, Vol 6: Fluid Mechanics, Section 130 (Pergamon, Oxford,1959).

[31] B. Carter, I.M. Khalatnikov, Phys. Rev. D45 (1992) 4536.

[32] B. Carter, Proc. Roy. Soc. Lond. A433 (1991) 45.

[33] N. Turok, Nucl. Phys. B242 (1984) 520.

[34] T. Vachaspati, A. Vilenkin, Phys. Rev. D31 (1985) 3035.

[35] R. Durer, Nucl. Phys. B328 (1989) 238.

[36] B. Carter, J. Geom. Phys. 8 (1992) 53

[37] B. Carter, Class. Qantum Grav. 9 (1992) 19.

[38] B. Carter, I.M. Khalatnikov, Ann. Phys. 219 (1992) 243.

[39] B. Carter, I.M. Khalatnikov, Rev. Math. Phys. 6, (1994) 277.

[40] B. Carter, "Covariant Theory of Conductivity in Ideal Fluid or Solid Media", in Relativistic Fluid Dynamics, ed. A.M. Anile, \& Y. Choquet-Bruhat, Lecture Notes in Mathematics 1385 (Springer - Verlag, Heidelberg, 1989) 1. 